\title{
Performance Evaluation of Dynamic QoS-Aware CAC (DQA-CAC) Algorithm for Broadband Networks
}

\author{
Solomon Orduen Yese, Abdulhakeem Abdulazeez, and Aminu Mohammed
}

\begin{abstract}
Call admission control (CAC) is a technique deployed in the management of network resources in mobile broadband networks. Despite its importance, the WiMAX technology like most mobile broadband networks does not make provision for $\mathrm{CAC}$, making it an open area of research. A dynamic QoS-Aware Call Admission Control (DQACAC) scheme was recently proposed to improve resource utilization and ensure QoS. Several simulation experiments were conducted to evaluate the performance of the DQA-CAC against other CAC algorithms. The results of the simulation indicated that the DQA-CAC outperformed the existing CAC schemes in terms of reduced new connection blocking rate. However, the evaluation failed to consider other performance metrics like resource utilization and throughput that are also very crucial in the real-world scenario. This paper therefore attempts to improve the performance of the DQA-CAC by investigating its performance in terms of additional metrics like the average system throughput and resource utilization that further mimic the real-world experience. A Java based discrete event simulator designed for the study was used and the simulation results indicate that the DQA-CAC algorithm outperforms the existing CAC algorithms in terms of improved per-flow throughput, average system throughput and resource utilization in addition to reducing the connection blocking rate.

Index Terms - adaptive bandwidth degradation; bandwidth reservation; mobile broadband networks; call admission control, performance evaluation.
\end{abstract}

\section{INTRODUCTION}

The current crop of mobile broadband network such as Worldwide Interoperability for Microwave Access (WiMAX) are tasked with support for integrated services

such as data, voice and multimedia with differentiated quality of service (QoS) [1][2][3]. Thus, making QoS provisioning one of the most important features required in mobile broadband networks. To maintain QoS, despite limited network resources, the number of simultanqdf6zSqeous network users need to be limited. However, limiting the number of users may end up upsetting another key feature of a good network i.e. efficient resource utilization [4]. Maintaining QoS while also ensuring efficient resource utilization is usually a herculean task.

A call admission control (CAC) algorithm is therefore

Published on November 16, 2019.

Solomon Orduen Yese, Computer Science Unit, Department of Mathematics, Usmanu Danfodiyo University Sokoto (UDUS), Nigeria.

(e-mails: yese.solomon@udusok.edu.ng)

Abdulhakeem Abdulazeez, Computer Science Unit, Department of Mathematics, UDUS Nigeria.

(email: abdulazeez.abdulhakeem@udusok.edu.ng)

Aminu Mohammed, Computer Science Unit, Department of

Mathematics, UDUS Nigeria.

(email: mohammed.aminu@udsok.edu.ng) needed to serve as a regulatory mechanism. The CAC algorithm decides whether or not a new connection request should be admitted or rejected depending on whether the new connection can be accepted and the QoS of existing connections will still be maintained [5]. Many CAC schemes have been proposed over the years [5-15] to ensure efficient resource utilization and or ensure QoS. More recently, a DQA-CAC was proposed in [15] with its performance evaluated against other schemes. The results indicate that the DQA-CAC outperforms the QA-CAC in terms of reduced new connection blocking rate. However, the evaluation of the schemes was performed without recourse to other key network parameters like resource utilization and throughput that is affected by the reduced blocking rate. Thus, the need to evaluate the performance of the scheme in terms of other QoS parameters like average system throughout, system resource utilization and per-flow throughput.

In this paper, a performance evaluation of the dynamic

QoS-Aware CAC algorithm earlier proposed is carried out against other schemes to determine the effect of the scheme on the resource utilization and throughput in addition to the blocking rate earlier determined.

The rest of the paper is organized as follows. Related call admission control algorithms are reviewed in Section II. The basic idea of Dynamic QoS-Aware CAC algorithm is presented in Section III. Section IV presents the performance comparison while the Section $\mathrm{V}$ concludes the paper.

\section{RELATED WORKS}

This section therefore presents a review of related works with a focus on the DQA-CAC.

The QoS-Aware CAC with BR and BD scheme was proposed in [5] to avoid starvation and improve resource utilization. The scheme introduces a new admission criterion according to service classes in order to increase the acceptance rate of connections into the system. The criteria also employ a BD policy to admit more users in the presence of inadequate resources. It also utilizes a dynamic threshold to tune the quantity of bandwidth reserved for HCs subject to the system load. The scheme accommodates more users as well as guarantees QoS to the different connection classes. However, it wastes resources due to fixed BD policy used that degrades resources from existing connections without recourse to the amount needed. Thus, prompting a call to be dropped when the degraded resources are not enough to admit the requesting call.

In [6-14], various CAC scheme have been proposed to either improve resource utilization or guarantee QoS. While these schemes have fulfilled their various objectives, they fail to create a balance by ensuring that the two core 
objectives of resource management (ensuring efficient resource utilization and QoS) are maintained without necessarily having to sacrifice one for the other. It is in response to this challenge, that the QA-CAC was proposed to both ensure QoS and improve resource utilization. However, the scheme also wastes resources. Consequently, the DQA-CAC was lately proposed in [15] to mitigate against this waste.

In [15], a proposed a dynamic QoS-Aware call admission control algorithm (DQA-CAC) to improve resources utilization. The DCAC admits real time connections (UGS, ertPS and rtPS) based on their maximum sustained traffic rate (MSTR) requirement but admits non-real time connections (nrtPS and BE) based on their minimum required traffic rate (MRTR). However, when resources are not sufficient to admit a new incoming connection, it employs a pre-degradation check mechanism to determine whether or not the resources to be mobbed from the existing ertPS and rtPS connections will be sufficient to meet the requirements of the requesting connection before going ahead to degrade and accept the connection. Else, the connection is rejected. The scheme reduces blocking and dropping rates.

\section{DYNAMIC QOS-AWARE CAC ALGORITHM}

This section presents the Dynamic QoS-Aware CAC algorithm.

The DQA-CAC algorithm adopts the same admission criterion as in [5]. That is, it admits UGS, ertPS and rtPS connections base on their MSTR requirements but admits the non-real time connections (nrtPS and BE) based on their MRTR requirements. However, when resources are insufficient, it employs a pre-bandwidth degradation technique that first determines whether the bandwidth acquired from degrading existing ertPS and rtPS connections will be sufficient to meet the bandwidth requested by the incoming connection. If the check is positive, degradation is performed on ertPS and rtPS connections in a stepwise manner, starting with the rtPS connection, else, the connection is rejected and the degradation is not performed. Also, the DQA-CAC deploys a dynamic degradation procedure based on a bandwidth intelligent function that ensures that the bandwidth degraded is not much in excess of what is needed by the requesting connection. The various algorithms are as presented in [15]

\section{PERFORMANCE EVALUATION}

The performance reevaluation of the DQA-CAC scheme against other schemes using a self-developed Java-based discrete event simulator. The schemes are evaluated based on three additional metrics: system resource utilization, average system throughput and per-flow throughput. The topology involves a single base station with several subscriber stations with same simulation parameters as used in [15] but with increased connection arrival rate of 0 to 0.7 as against 0 to 0.45 previously used. The non-inclusion of handoff scenario and non-evaluation of the handoff dropping rate is because both schemes use the same handoff reservation mechanism and are bound to have similarly performance in terms of dropping of handoff connections. The assumed data rates for this simulation are as presented in table 1 .

Table 1 Parameters for IEEE 802.16e PHY layer data rates

\begin{tabular}{|c|c|}
\hline Parameter & Value (kbps) \\
\hline$B_{U G S}^{M S T R}, B_{U G S}^{M R T R}$ & 256,256 \\
\hline$B_{\text {ertPS }}^{M S T R}, B_{\text {ertPS }}^{M R T R}$ & 1024,512 \\
\hline$B_{r t P S}^{M S T R}, B_{r t P S}^{M R T R}$ & 1024,512 \\
\hline$B_{n r t P S}^{M S T R}, B_{n r t P S}^{M R T R}$ & 1024,512 \\
\hline$B_{B E}^{M S T R}, B_{B E}^{M R T R}$ & 1024,1024 \\
\hline
\end{tabular}

The arrival of the new connections is assumed to follow a random distribution. This is to enable the system mimic the real network scenario as much as possible.

\section{A. Connection Blocking Rate}

Figure 1 shows the connection blocking rate for the DQA and QA CAC schemes. The result indicates that while the QA starts dropping of packets at 0.2 arrival rate, the DQA only starts blocking of packets at 0.24 . The results also indicate that as the connection arrival rate increases, the DQA performs better than the QA in that it blocks new connections at a slower rate when compared to the QA. This improved performance is due to the pre-bandwidth degradation mechanism that checks the appropriate amount of resources to be degraded and the dynamic degradation mechanism that prevents unnecessary degradation of the existing connections.

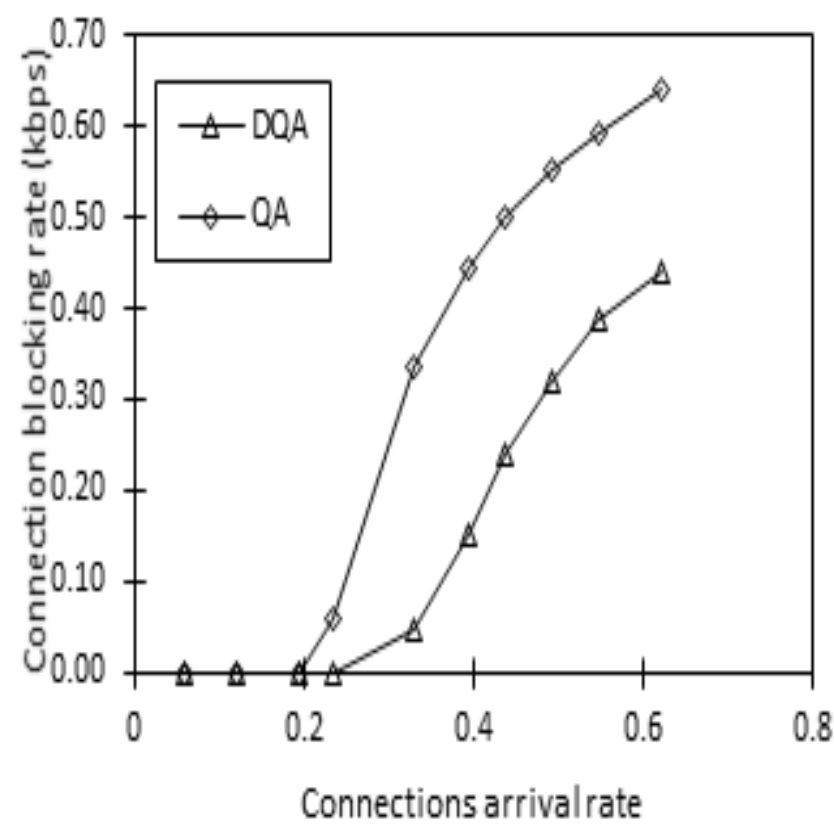

Fig. 1 Blocking rates of the two schemes

\section{B. Average System Utilization}

Figure 2 shows the average system throughput for the DQA and QA CAC schemes. The result indicates that while the network is underutilized, the performance of both schemes is similar. However, when the arrival rate increases, the DQA outperforms the QA as the throughput continuously increases. This improvement in throughput 
performance is due to pre-check mechanism that ensures that degradation is only performed when it is determined that the degraded resources can meet the demands of the requesting connection and the intelligent bandwidth degradation mechanism that ensures that only the required of bandwidth is degraded.

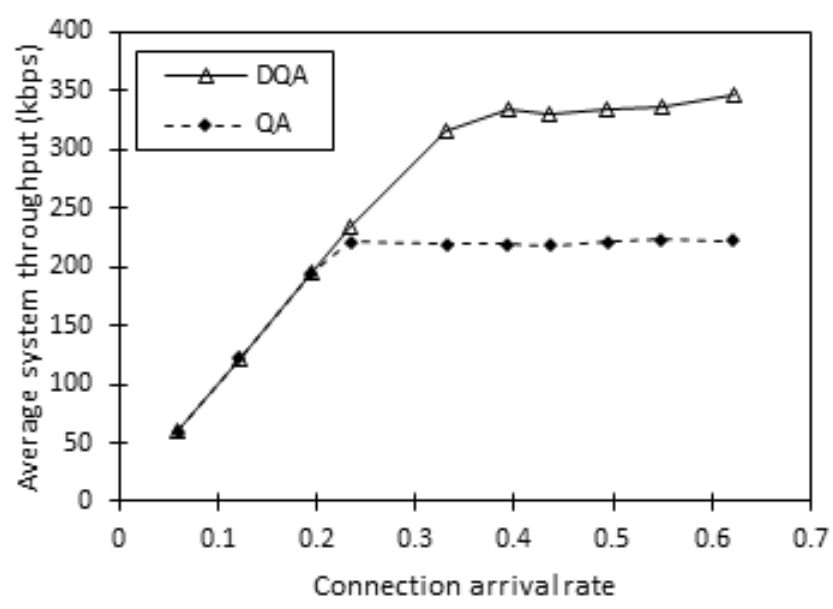

Fig. 2 Average System throughput of the two schemes

\section{Average Resource Utilization}

Figure 3 shows the average resource utilization for the DQA and QA CAC schemes. The result indicates that the DQA outperforms the QA as it improves resources utilization in the midst of increasing arrival rate of connections. This improvement in resource utilization can be traced to the pre-check mechanism that ensures that degradation is only performed when it is determined that the degraded resources can meet the demands of the requesting connection and the intelligent bandwidth degradation mechanism that ensures that only the required of bandwidth is degraded thus, maintaining the bandwidth utilization of system when degradation is not necessary.

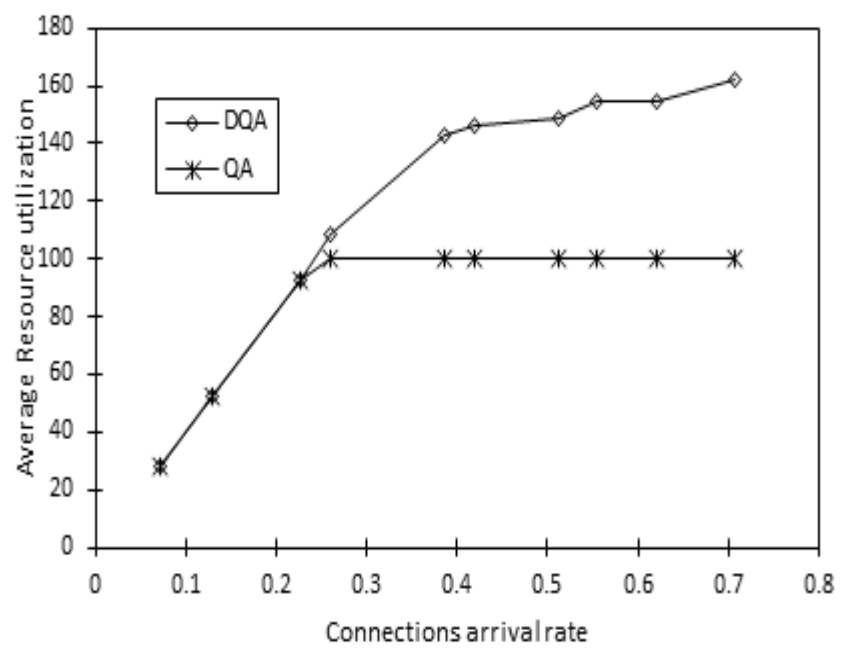

Fig. 3 Resource Utilization of the two schemes

\section{Per-flow Throughput}

Figure 4 shows the throughput of UGS traffic class for QA-CA and the proposed Dynamic QoS-Aware CAC scheme. It shows that when the network arrival rate is low, i.e., from $0.05-0.25$, both schemes have similar throughput because the bandwidth has not been saturated. But as the network arrival rate increases, the proposed scheme performs better than the QA-CAC scheme. Also, zigzag nature of the graph can be attributed to the random generation of packets which is done in order to mimic reallife network scenarios. The increase in the throughput is attributed to the new pre-check mechanism and improved degradation algorithm that ensures that degradation is performed if and only the estimated bandwidth to be gotten from the degradation will be sufficient to admit the incoming connection request and that only the needed bandwidth is degraded at a time respectively. Thus, increasing the departure rate and freeing more bandwidth for the admission of more connections into the network.

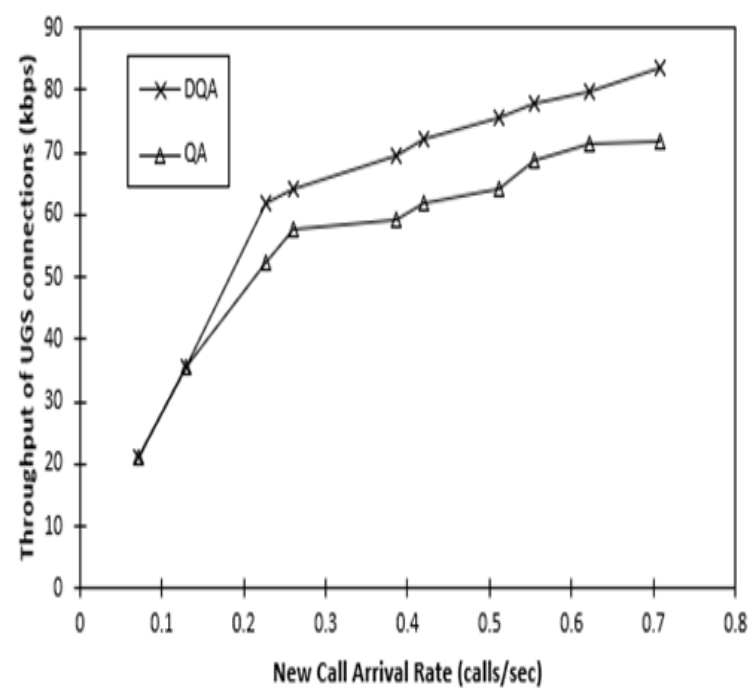

Fig. 4: Throughput of UGS traffic class of the two schemes

Figure 5 shows the throughput of ertPS traffic class for QA-CA and the proposed Dynamic QoS-Aware CAC scheme. It shows that when the network arrival rate is low, i.e., from $0.05-0.23$, both schemes have similar throughput because the bandwidth has not been saturated. But as the network arrival rate increases, the proposed scheme performs better than the QA-CAC scheme. Also, zigzag nature of the graph can be attributed to the random generation of packets which is done in order to mimic reallife network scenarios. The increase in the throughput is attributed to the new pre-check mechanism and improved degradation algorithm that ensures that degradation is performed if and only the estimated bandwidth to be gotten from the degradation will be sufficient to admit the incoming connection request and that only the needed bandwidth is degraded at a time respectively. Thus, increasing the departure rate and freeing more bandwidth for the admission of more connections into the network. 


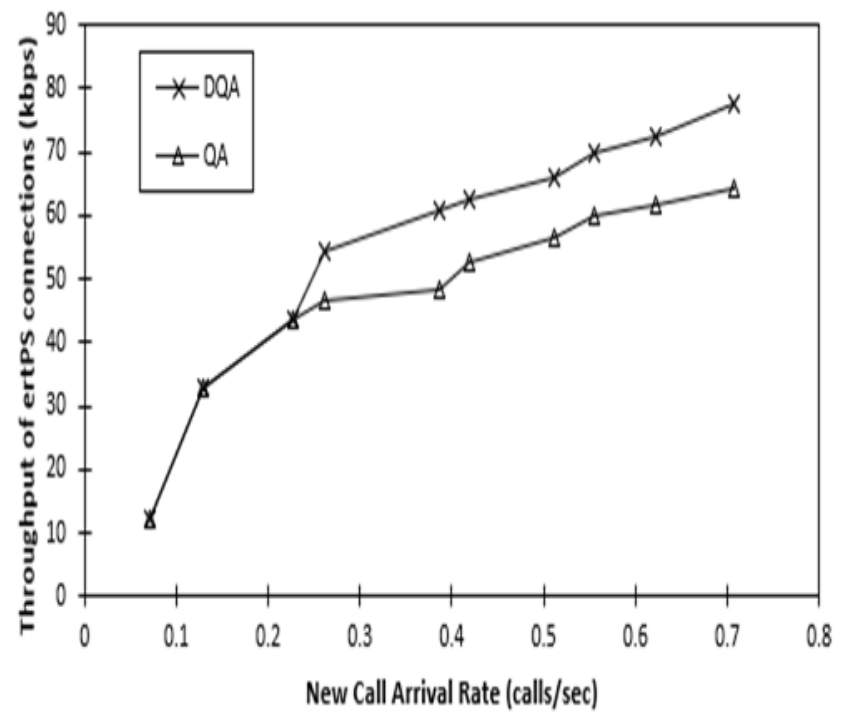

Fig. 5: Throughput of ertPS traffic class of the two schemes

Figure 6 shows the throughput of rtPS traffic class for QA-CA and the proposed Dynamic QoS-Aware CAC scheme. It shows that when the network arrival rate is low, i.e., from $0.05-0.25$, both schemes have similar throughput because the bandwidth has not been saturated. But as the network arrival rate increases, the proposed scheme performs better than the QA-CAC scheme. Also, zigzag nature of the graph can be attributed to the random generation of packets which is done in order to mimic reallife network scenarios. The increase in the throughput is attributed to the new pre-check mechanism and improved degradation algorithm that ensures that degradation is performed if and only the estimated bandwidth to be gotten from the degradation will be sufficient to admit the incoming connection request and that only the needed bandwidth is degraded at a time respectively. Thus, increasing the departure rate and freeing more bandwidth for the admission of more connections into the network.

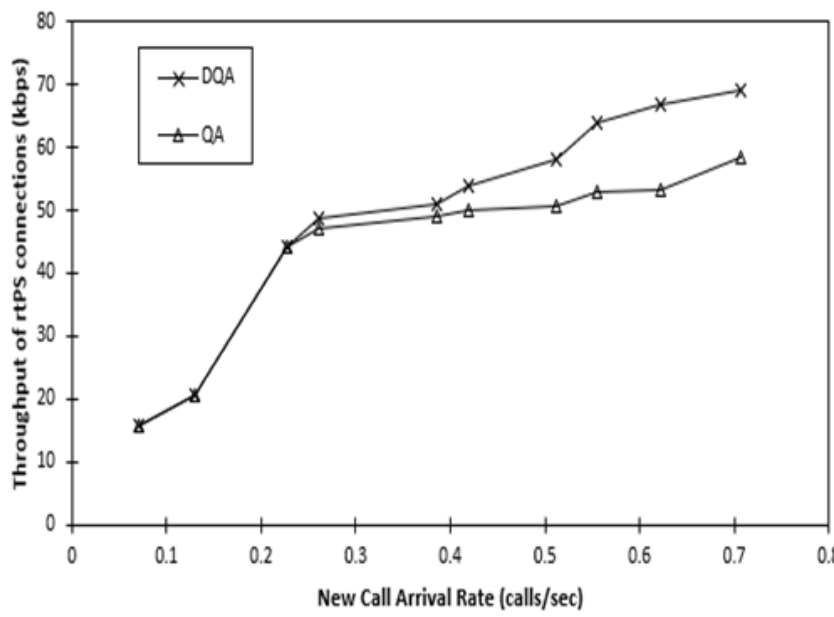

Fig. 6: Throughput of rtPS traffic class of the two schemes

Figure 7 shows the throughput of nrtPS traffic class for QA-CA and the proposed Dynamic QoS-Aware CAC scheme. It shows that when the network arrival rate is low, i.e., from $0.05-0.24$, both schemes have similar throughput because the bandwidth has not been saturated. But as the network arrival rate increases, the proposed scheme performs better than the QA-CAC scheme. Also, the zigzag nature of the graph can be attributed to the random generation of packets which is done in order to mimic reallife network scenarios. The increase in the throughput is attributed to the new pre-check mechanism and improved degradation algorithm that ensures that degradation is performed if and only the estimated bandwidth to be gotten from the degradation will be sufficient to admit the incoming connection request and that only the needed bandwidth is degraded at a time respectively. Thus, increasing the departure rate and freeing more bandwidth for the admission of more connections into the network.

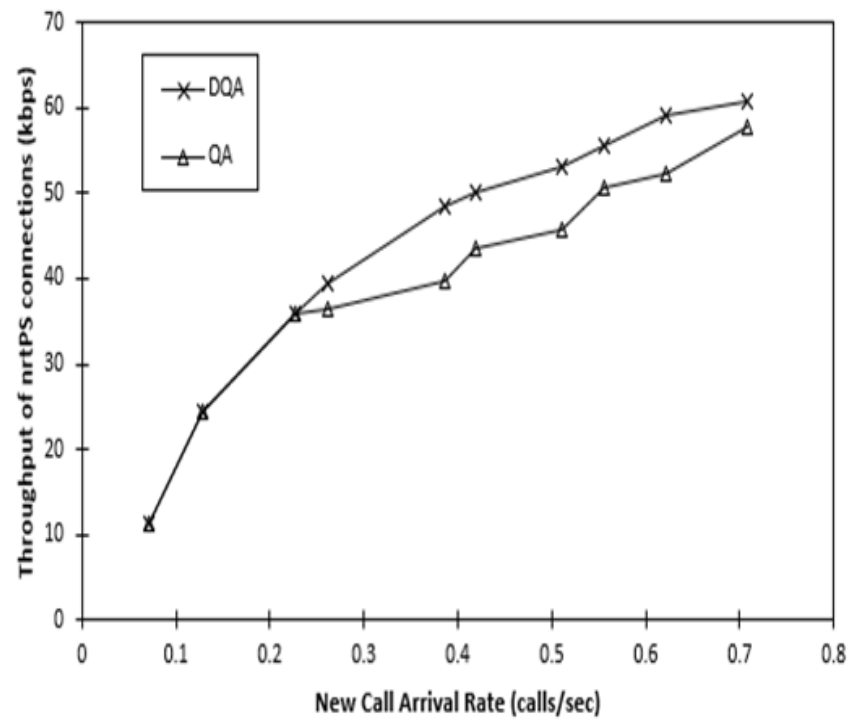

Fig. 7: Throughput of nrtPS traffic class of the two schemes

Figure 8 shows the throughput of BE traffic class for QA$\mathrm{CA}$ and the proposed Dynamic QoS-Aware CAC scheme. It shows that when the network arrival rate is low, i.e., from $0.05-0.26$, both schemes have similar throughput because the bandwidth has not been saturated. But as the network arrival rate increases, the proposed scheme performs better than the QA-CAC scheme. Also, the zigzag nature of the graph can be attributed to the random generation of packets which is done in order to mimic real-life network scenarios. The increase in the throughput is attributed to the new precheck mechanism and improved degradation algorithm that ensures that degradation is performed if and only the estimated bandwidth to be gotten from the degradation will be sufficient to admit the incoming connection request and that only the needed bandwidth is degraded at a time respectively. Thus, increasing the departure rate and freeing more bandwidth for the admission of more connections into the network. 


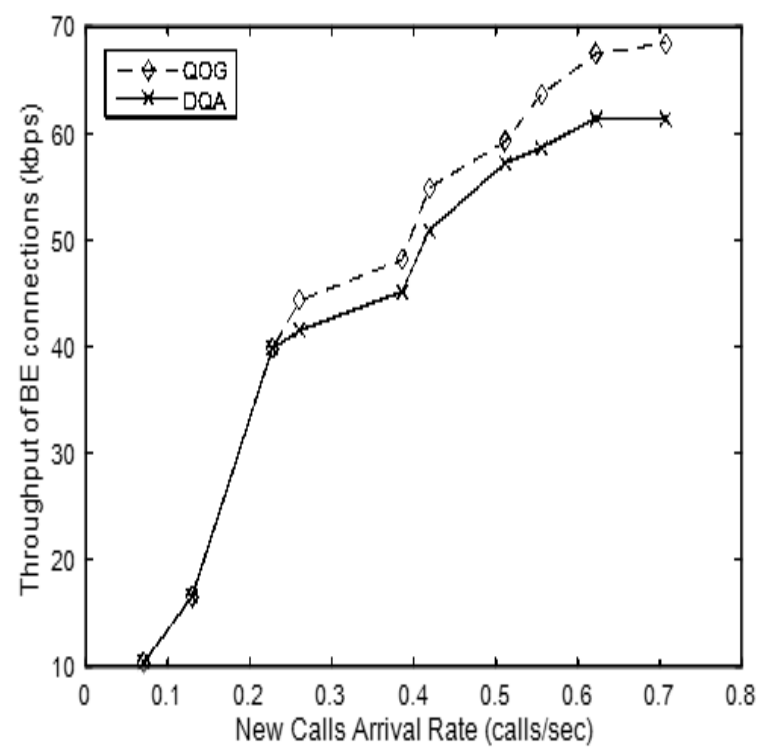

Fig. 8: Throughput of BE traffic class of the two schemes

\section{CONCLUSION}

The paper presents a performance evaluation of the Dynamic QoS-Aware CAC (DQACAC) scheme against the QoS-Aware CAC (QA-CAC) schemes to further determine the viability of the DQA-CAC scheme. It evaluates the performance of the two schemes using additional performance metrics like the average system throughput and the system utilization as well as per-flow throughput in additional to the new connection blocking rate earlier evaluated. The results of the further simulations indicate that despite the increased number of subscriber stations, the DQA-CAC still outperforms the QA-CAC in all evaluated areas. That is, in addition to reducing the new connection blocking rate, the DQA-CAC also increases the average throughput of the system and the resource utilization of the system as well as the per-flow throughput.

\section{ACKNOWLEDGMENT}

The authors will like to heartily appreciate the Tertiary Education Trust Fund (TETFund) Nigeria for supporting this research.

\section{REFERENCES}

[1] S. Pathak and S. Batra, "Next generation 4G WiMAX networksIEEE 802.16 standard”. Sundayapandian et al. (Eds): CoNeCo, WiMo, NLP, CRYPSIS, ICAIT, ICDIP, ITCE, CS \& IT, 7, (2012), pp. 507-518.

[2] A.L. Yadav, P. Vyavahare, and P. Bansod. QoS in WiMAX hybrid schedulers for heterogeneous traffic and their performance comparison. International Journal of Electronics, (2019). pp.1-15.

[3] A.A. Ehsan, Quality of Service Support in IEEE 802.16 Broadband Wireless Access Networks (Master's Thesis, Monash University Malaysia, Australia) (2006). Unpublished.

[4] Afzali M, AbuBakar K, Lloret J. Adaptive Resource Allocation for WiMAX Mesh Network. Wireless Personal Communications. 2019:1-9.

[5] I. Saidu, S. Subramaniam, A. Jaafar and Z. A. Zukarnain, "A QoS-Aware CAC with $B$ and width Reservation and Degradation Scheme in IEEE 802.16e Networks", Wireless PersCommun (2015), 82(4), pp. 2673-2693.

[6] Z. Patel and U. Dalal, "Improved CAC Scheme for WiMAX with Adaptive Bandwidth Reservation and Degradation Policy". Int'l Journal of Computing, Communications \& Instrumentation Eng. (IJCCIE), 2015, 2(2), pp. 144-149.

[7] C. Wang, W. Yan and H. Lo, "Dynamic Admission Control and Bandwidth Reservation for IEEE 802.16e mobile WiMAX networks, EURASIP Journal on Wireless Communications and Networking, 2012, pp. 1-20.

[8] L. Wang, F. Liu, Y. Ji, and N. Ruangchaijatupon "Admission control for non-preprovisioned service flow in wireless metropolitan area networks", In Universal Multiservice Networks, ECUMN'07. Fourth European Conference. (2007). pp.243-249.

[9] J.S. Ganesh and P.T.V. Bhuvaneswari, "Enhanced Call Admission Control for WiMAX networks", In Recent Trends in Information Technology (ICRTIT), International Conference, Chennai (2011, June). pp. 33-36.

[10] D.S. Shúaibu, S. Yusof, N. Fisal, S. H. S. Ariffin, R. A. Rashid and Y. S. Baguda, "Call admission control in mobile WiMAX IEEE802.16e with dual partitioning”. In 2010 International conference on intelligent network and computing (ICINC), Kuala Lampur, Malaysia (2010), vol. 2, pp. 21-24.

[11] A. Antonopoulos, C. Skianis, and C. Verikoukis, "Traffic-aware connection admission control scheme for broadband mobile systems". In Global Telecommunications Conference (GLOBECOM 2010), 2010 IEEE (pp. 1-5), IEEE.

[12] R. M. Prasad, "An efficient connection admission control mechanism for IEEE 802.16e networks". Global Journal of Computer Science and Technology (2010). 10(8), pp. 97-101.

[13] D. S. Shu'aibu, S. Yusof, N. Fisal, S. H. S. Ariffin, R. A. Rashid, N. M. AbdulLatiff and Y. S. Baguda, "Fuzzy logic partitionbased call admission control for mobile WiMAX", ISRN Communications and Networking (2011). pp.1-10.

[14] C. Wang and K.Y. Chan, "Utility-based admission control for mobile WiMAX networks", Wireless Networks (2013)., 19(2), pp. 207-218.

[15] A. Mohammed, I. Saidu, Y. Solomon, and B. Y. Isah. A dynamic QoS-aware call admission control algorithm for mobile broadband networks. International Conference on Computing Networking and Informatics (ICCNI), 2017 IEEE (pp. 1-5) 